\title{
Thienyl Sidechain Substitution and Backbone Fluorination of Benzodithiophene-based Donor Polymers Concertedly Minimize Carrier Losses in ITIC-based Organic Solar Cells
}

\author{
Jafar I. Khan*, Yuliar Firdaus, Federico Cruciani, Shengjian Liu, Thomas Anthopoulos, Pierre \\ M. Beaujuge, and Frédéric Laquai*
}

\begin{abstract}
Dr. J. I. Khan, Dr. Y. Firdaus, Dr. Federico Cruciani, Dr. Shengjian Liu, Prof. T. Anthopoulos, Ass. Prof. P. M. Beaujuge, Assoc. Prof. F. Laquai
\end{abstract}

\author{
King Abdullah University of Science and Technology (KAUST) \\ KAUST Solar Center (KSC) \\ Division of Physical Sciences and Engineering (PSE) \\ Thuwal 23955-6900, Kingdom of Saudi Arabia \\ E-mail: \\ jafar.khan@kaust.edu.sa \\ frederic.laquai@kaust.edu.sa
}




\begin{abstract}
Non-fullerene acceptor (NFA) based organic solar cells have outperformed fullerene-based devices, yet their photophysics is less well understood. Herein, changes in the donor polymer backbone side-chain substitution and backbone fluorination in benzodithiophene (BDT)-thiophene copolymers are linked to the photophysical processes and performance of bulk heterojunction (BHJ) solar cells, using ITIC as NFA. Increased geminate recombination is observed when the donor polymer is alkoxy-substituted in conjunction with faster non-geminate recombination of free charges, limiting both the short circuit current and device fill factor. In contrast, thienylsubstitution reduces geminate recombination, albeit non-geminate recombination remains significant, leading to improved short circuit current density, yet not fill factor. Only the combination of thienyl-substitution and polymer backbone fluorination yields both efficient charge separation and significantly reduced non-geminate recombination, resulting in fill factors (FFs) in excess of $60 \%$. Time-delayed collection field measurements ascertain that charge generation is field-independent in the thienyl-substituted donor polymer:ITIC systems, while weakly field dependent in the alkoxy-substitued polymer:ITIC blend, indicating the low FFs are primarily caused by non-geminate recombination. This work provides insight into the interplay of donor polymer structure, BHJ photophysics, and device performance for a prototypical NFA, namely ITIC. More specifically, it links the donor polymer chemical structure to quantifiable changes of kinetic parameters and the yield of individual processes in ITIC-based BHJ blends.
\end{abstract}

Keywords: ultrafast spectroscopy, non-fullerene acceptor, organic photovoltaics, bulk heterojunction, charge generation 


\section{Introduction}

Non-fullerene acceptors (NFAs) have shown significant potential as next generation acceptors in organic photovoltaics (OPV). The first NFA that outperformed the commonly-used fullerene was 3,9-bis(2-methylene-(3-(1,1-dicyanomethylene)-indanone))-5,5,11,11-tetrakis(4-hexylphenyl)dithieno[2,3-d:2',3'-d']-s-indaceno[1,2-b:5,6-b']dithiophene (ITIC). ${ }^{1}$ Since then, the power conversion efficiency (PCE) of NFA-OPV has reached values in excess of $14 \%$, when using the fluorinated ITIC-derivative, IT-4F. ${ }^{2,3}$ Recently, PCEs between 15-18 \% have been achieved using electron-deficient core ladder-type NFA acceptors. ${ }^{4-9}$

The rapid development of NFA-based OPV is not only driven by the development of novel NFAs, but also by new donor molecules, precisely matched in energetics, yielding 'ideal' BHJ morphology. However, designing the best combination of an NFA and donor material to obtain high-efficiency bulk heterojunction (BHJ) solar cells is largely a challenging and multifaceted endeavor. For example, the substitution pattern of the donor polymer influences the BHJ solar cell efficiencies. ${ }^{10}$ Prior studies have demonstrated that the presence/absence of polar groups, such as fluorine and ring substituents along the polymer backbone significantly affects the device performance. ${ }^{10-22}$ Molecular dynamics simulations and solid-state NMR experiments revealed that fluorination leads to increased electronic couplings between neighboring chains. ${ }^{23}$ Additionally, fluorine substituents lead to a preferential face-on alignment of polymer chains with respect to the carrier-extracting electrodes, beneficial for carrier extraction which competes with charge recombination in the thin film. ${ }^{24,25}$ On the other hand, ring substituents (such as furan, thiophene, and selenothiophene) incorporated in side chains attached to the polymer donors can induce significant morphological effects in BHJs. ${ }^{15}$ Hou et al. investigated the effect of thienyl-chain substitution of benzo[1,2-b:4,5-b']dithiophene (BDT) on the photovoltaic properties of the 
polymers. ${ }^{26,27}$ They found that polymers with thienyl-substituted BDT (so called 2-dimensional polymers) exhibit improved thermal stabilities, red-shifted absorption spectra, decreased highest occupied molecular orbital (HOMO) and lowest unoccupied molecular orbital (LUMO) energy levels, and increased hole mobility, altogether leading to improved photovoltaic properties in comparison to alkoxy-substituted analogues. A recent work by Firdaus et al. examined a set of wide-bandgap polymer donor analogues composed of benzo[1,2-b:4,5- $\left.b^{\prime}\right]$ dithiophene (BDT), and thienyl $([2 \mathrm{H}] \mathrm{T})$ or 3,4-difluorothiophene $([2 \mathrm{~F}] \mathrm{T})$ motifs, and their BHJ device performance when combined with ITIC. ${ }^{10}$ The work concluded that the fluorine- and ring-substituted polymer PBDT(T)[2F]T largely outperforms two structurally-related polymer donors, reaching power conversion efficiencies (PCE) as high as $9.8 \%$.

Here, we reveal the photophysical processes in BHJ solar cells comprised of either PBDT[2H]T, $\operatorname{PBDT}(\mathrm{T})[2 \mathrm{H}] \mathrm{T}$, or PBDT(T)[2F]T as donor polymer when combined with the prototypic NFA, ITIC. Specifically, we reveal the impact of alkoxy vs. thienyl substitution of the polymer backbone and of backbone fluorination on the yield of individual photophysical processes, kinetic parameters, and field-dependence of charge separation, and thereby their impact on PCE. We infer that thienyl sidechains and backbone fluorination concertedly increase the short circuit current density. Furthermore, while fill factors $(F F \mathrm{~s})$ are limited to $\sim 40 \%$ for the alkoxy and thienylsubstituted polymers, they are improved to $60 \%$ upon backbone fluorination. Transient absorption (TA) and time-resolved photoluminescence (TR-PL) spectroscopy are used to interrogate the origin of the BHJ solar cell performance differences, ${ }^{28}$ specifically to quantify the efficiencylimiting photophysical processes. Incomplete exciton dissociation and enhanced geminate recombination $(\sim 20 \%)$ are found in PBDT[2H]T:ITIC in contrast to PBDT(T)[2F]T:ITIC (8\%), and PBDT(T)[2H]T:ITIC (14\%). Time-delayed collection field (TDCF) measurements indicate 
field independent charge generation in PBDT(T)[2F]T:ITIC and PBDT(T)[2H]T:ITIC blends and only weakly field-dependent generation in PBDT[2H]T:ITIC, indicating that FFs in all systems are primarily governed by non-geminate recombination competing with charge extraction. Finally, drift-diffusion simulations of the devices' J-V characteristics demonstrate a good agreement between the experimentally-measured device $\mathrm{J}-\mathrm{V}$ curves and the simulated ones based on the kinetic parameters and efficiencies determined from spectroscopic measurements.

\section{Experimental Methods}

Device Fabrication: The solar cells were prepared on glass substrates with tin-doped indium oxide (ITO, $15 \Omega \mathrm{sq}^{-1}$ ) patterned on the surface (device area: $0.1 \mathrm{~cm}^{2}$ ). The ITO were cleaned by sequential ultra-sonication in dilute Extran 300 detergent solution, deionized water, acetone, and isopropyl alcohol for 20 minutes each. The substrates were then cleaned by UV-ozone treatment for 30 minutes. A thin layer $(\sim 25 \mathrm{~nm})$ of amorphous $\mathrm{ZnO}$ was spin-cast onto the UV-treated samples, dried on a hot plate at $150{ }^{\circ} \mathrm{C}$ for 20 minutes, and afterwards the substrates were transferred into a dry nitrogen glovebox. The active-layers were prepared by dissolving the polymers and ITIC in chlorobenzene (20 $\mathrm{mg} / \mathrm{ml}$ concentration) using polymer:ITIC ratios of 1:1. The polymer:ITIC blend solutions were stirred overnight at $110{ }^{\circ} \mathrm{C}$ and then the temperature of the mixtures was lowered to $90{ }^{\circ} \mathrm{C}$ prior to solution-casting onto the substrates. The active layers were spin-cast from the solutions at $90{ }^{\circ} \mathrm{C}$ at an optimized speed of $1500 \mathrm{rpm}$ in a time period of 30 s, using a programmable spin-coater from Specialty Coating Systems (Model G3P-8), resulting in films of 65 to $80 \mathrm{~nm}$ in thickness. Next, the samples were placed in a thermal evaporator for evaporation of 7-nm thick layers of $\mathrm{MoO}_{3}$ deposited at a rate of $0.3 \mathrm{As}^{-1}$, and 100-nm thick silver 
electrodes deposited at a rate of 3-4 $\AA \mathrm{s}^{-1}$; pressure of less than $2 \times 10^{-6}$ Torr. Following electrode deposition, samples underwent $\mathrm{J}-\mathrm{V}$ testing.

UV-Vis-spectroscopy: Steady-state absorption measurements were performed using a Cary 5000 UV-visible spectrometer (Agilent Technologies).

Device characterization: J-V measurements of solar cells were performed in a $\mathrm{N}_{2}$ filled glovebox using a Keithley 2400 source meter and an Oriel Sol3A Class AAA solar simulator calibrated to 1 sun, AM1.5G, with a KG-5 silicon reference cell certified by Newport. EQE was characterized using a specially designed EQE system (PV measurement Inc.). The Measurements were performed at zero bias by illuminating the device with monochromatic light supplied from a Xenon arc lamp in combination with a dual-grating monochromator. The number of photons incident on the sample was calculated for each wavelength by using a silicon photodiode calibrated by NIST. The IQE spectra were calculated from EQE spectra using the relation: $I Q E=E Q E /(1-$ Reflectance - Parasitic Absorption $)$. The reflectance spectra were collected with the integrating sphere using the same EQE system while the parasitic absorption spectra were obtained from transfer matrix modelling.

Ultrafast time-resolved measurements: Transient absorption (TA) spectroscopy was carried out using a home-built pump-probe setup. Two different configurations of the setup were used for either short delay, namely 100 fs to 8 ns experiments, or long delay, namely 1 ns to $100 \mu$ s delays, as described below:

The output of titanium:sapphire amplifier (Coherent LEGEND DUO, $4.5 \mathrm{~mJ}, 3 \mathrm{kHz}, 100 \mathrm{fs}$ ) was split into three beams $(2 \mathrm{~mJ}, 1 \mathrm{~mJ}$, and $1.5 \mathrm{~mJ})$. Two of them were used to separately pump two 
optical parametric amplifiers (OPA) (Light Conversion TOPAS Prime). The TOPAS 1 generates tunable pump pulses, while the TOPAS 2 generates signal $(1300 \mathrm{~nm})$ and idler $(2000 \mathrm{~nm})$ only. A fraction of the output signal of titanium:sapphire amplifier was focused into a c-cut $3 \mathrm{~mm}$ thick sapphire window, thereby generating a white-light supercontinuum from 500 to $1600 \mathrm{~nm}$. For short delay TA measurements, we used the TOPAS 1 for producing pump pulses while the probe pathway length to the sample was kept constant at approximately 5 meters between the output of the TOPAS1 and the sample. The pump pathway length was varied between 5.12 and $2.6 \mathrm{~m}$ with a broadband retroreflector mounted on automated mechanical delay stage (Newport linear stage IMS600CCHA controlled by a Newport XPS motion controller), thereby generating delays between pump and probe from -400 ps to $8 \mathrm{~ns}$. For measuring TA in the whole range of 500-1600 $\mathrm{nm}$, we used $800 \mathrm{~nm}$ to produce the white-light super continuum.

For the $1 \mathrm{~ns}$ to $100 \mu$ s delay (long delay) TA measurement, the same probe white-light supercontinuum as for the 100 fs to $8 \mathrm{~ns}$ delays. But the excitation light (pump pulse) was provided by an actively Q-switched Nd:YVO 4 laser (INNOLAS picolo AOT) frequency-doubled to provide pulses at $532 \mathrm{~nm}$. The laser was triggered by an electronic delay generator (Stanford Research Systems DG535), itself triggered by the TTL sync from the Legend DUO, allowing control of the delay between pump and probe with a jitter of roughly $100 \mathrm{ps.}$

Pump and probe beams were guided to the sample with reflective optics. The sample was kept under a dynamic vacuum of $<10^{-5} \mathrm{mbar}$. The transmitted fraction of the white light was guided to a custom-made prism spectrograph (Entwicklungsbüro Stresing) where it was dispersed by a prism onto a 512 pixel NMOS linear image sensor (HAMAMATSU S8381-512). The probe pulse repetition rate was $3 \mathrm{kHz}$, while the excitation pulses were mechanically chopped to $1.5 \mathrm{kHz}$ (100 fs to $8 \mathrm{~ns}$ delays) or directly generated at $1.5 \mathrm{kHz}$ frequency ( $1 \mathrm{~ns}$ to $100 \mu$ s delays), while the 
detector array was read out at $3 \mathrm{kHz}$. Adjacent diode readings corresponding to the transmission of the sample after excitation and in the absence of an excitation pulse were used to calculate $\Delta \mathrm{T} / \mathrm{T}$. Measurements were averaged over several thousand shots to obtain a good signal-to-noise ratio. The chirp induced by the transmissive optics was corrected with a home-built Matlab code by revaluating for each wavelength the delay at which pump and probe are simultaneously arriving on the sample as the time of the signal amplitude.

Time-delayed collection field: The home-built TDCF setup uses the second harmonic (532 nm) of an actively Q-switched sub-ns Nd: $\mathrm{YVO}_{4}$ laser (INNOLAS picolo AOT) operating at $5 \mathrm{kHz}$ as excitation with a pulse length of 0.7 ns. To minimize the RC response time (around 2 nanoseconds), a small device area of $1 \mathrm{~mm}^{2}$ is used. The samples were measured under dynamic vacuum conditions to avoid any degradation. A Keysight S1160A functional generator was used to provide the pre-bias and extraction bias, while a Keysight four channel digital oscilloscope was used to measure the current response of the device.

Time-resolved photoluminescence spectroscopy (TR-PL): For TR-PL experiments samples were excited with the wavelength-tunable output of an OPO (Radiantis Inspire HF-100), itself pumped by the fundamental of a Ti:sapphire fs-oscillator (Spectra Physics MaiTai eHP) at $820 \mathrm{~nm}$. The repetition rate of the fs pulses was adjusted by a pulse picker (APE Pulse Select). Typical pulse energies were in the range of several nJ. The PL of the samples was collected by an optical telescope (consisting of two plano-convex lenses) and focused on the slit of a spectrograph (PI Spectra Pro SP2300) and detected with a Streak Camera (Hamamatsu C10910) system with a temporal resolution of 1.4 ps. The data was acquired in photon counting mode using the Streak Camera software (HPDTA) and exported to Origin Pro 2017 for further analysis. 


\section{Results and Discussion}

\section{Materials and Device Performance}

a)

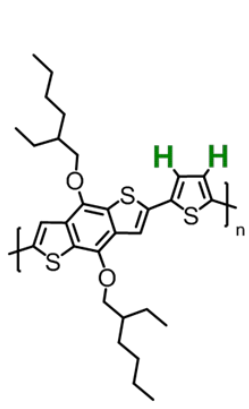

PBDT[2H]T

c)

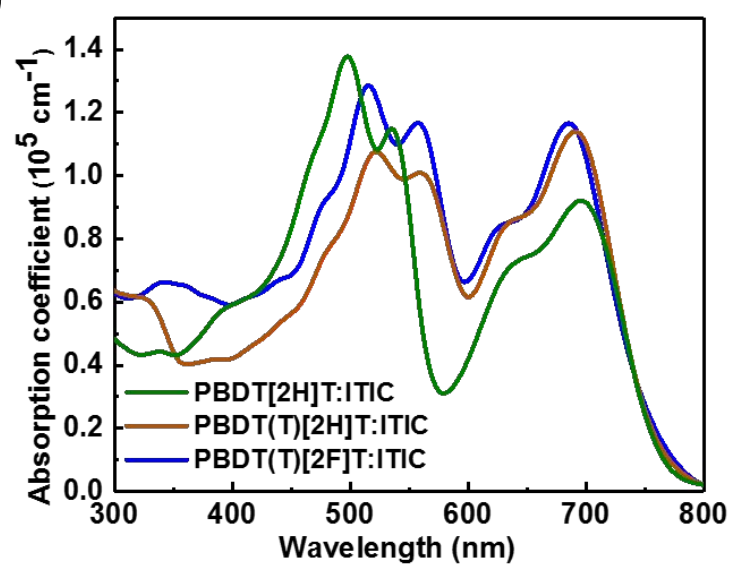

b)

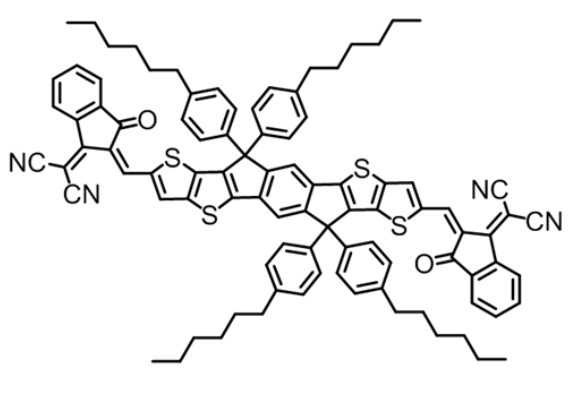

ITIC

Figure 1: a) Chemical structures of the donor polymers $\operatorname{PBDT}[2 \mathrm{H}] \mathrm{T}, \operatorname{PBDT}(\mathrm{T})[2 \mathrm{H}] \mathrm{T}$, and PBDT(T)[2F]T and b) of the non-fullerene acceptor ITIC. c) Absorption coefficient spectra of performance-optimized polymer:ITIC blends. d) J-V characteristics of the respective BHJ devices measured under one sun irradiance and the associated PCE values.

The chemical structures of the donor polymers $\operatorname{PBDT}[2 \mathrm{H}] \mathrm{T}, \mathrm{PBDT}(\mathrm{T})[2 \mathrm{H}] \mathrm{T}$, and $\mathrm{PBDT}(\mathrm{T})[2 \mathrm{~F}] \mathrm{T}$, designed and synthesized by us, and of the commercial ITIC NFA are presented in Figure 1a and $1 \mathrm{~b}$, respectively. The main differences in the polymer structures are the presence of thienyl-based sidechains in PBDT(T)[2H]T compared to alkoxy sidechains in PBDT[2H]T and both thienylbased sidechains and backbone fluorination and in PBDT(T)[2F]T. The absorption coefficients of 
thin films of the polymer:ITIC blends optimized for solar cell performance are depicted in Figure 1c. From Figure 1c and the UV-Vis absorbance spectra (Figure S1) it is discerned that all three polymers absorb strongly in the visible spectral range of $400-600 \mathrm{~nm}$, while the ITIC NFA absorbs in a complementary spectral range of $550-750 \mathrm{~nm}$ with an absorption maximum at $700 \mathrm{~nm}$. Consequently, the BHJ blends exhibit broad absorption spectra covering a range up to $\sim 770 \mathrm{~nm}$. BHJ solar cells with inverted structures, i.e., ITO/a-ZnO/Polymer donor:ITIC/ $\mathrm{MoO}_{3} / \mathrm{Ag}$ (device area: $\left.0.1 \mathrm{~cm}^{2}\right)$, were fabricated and tested under AM1.5G solar illumination $\left(100 \mathrm{~mW} \cdot \mathrm{cm}^{-2}\right)$. The PBDT $[2 \mathrm{H}] \mathrm{T}$ - and PBDT(T)[2F]T-based cells were fabricated according to the optimized conditions reported in our previous work. ${ }^{10}$ Optimized PBDT(T)[2H]T:ITIC (1:1 w/w) blend BHJ devices, not reported previously, were spin-cast from CB (cf. details in the Experimental Section). Figure 1d shows the current density-voltage (J-V) characteristics of optimized PBDT[2H]T, PBDT(T)[2H]T, and PBDT(T)[2F]T-based solar cells with ITIC as electron acceptor, and Table 1 provides the figures-of-merit of these devices. The PBDT[2H]T-based cells deliver a maximum PCE up to $2.5 \%$, with low short circuit current-density $\left(J_{\mathrm{SC}}\right)$ and fill-factor $(F F)$ values in the order of $7.3 \mathrm{~mA} \mathrm{~cm}^{-2}$ and $41 \%$ (Table 1), respectively. In contrast, the BHJ solar cells made of the thienyl-substituted polymer PBDT(T)[2H]T deliver higher $J_{\mathrm{SC}}$ of $13.8 \mathrm{~mA} \mathrm{~cm}^{-2}$, while the open circuit voltage $\left(V_{\mathrm{OC}}\right)$ of $0.72 \mathrm{~V}$ and the $F F$ of $40 \%$ are comparable to the donor polymer PBDT(T)[2H]T-based cells, resulting in an overall higher PCE of $4.2 \%$. In contrast, BHJ devices made of PBDT(T)[2F]T as donor polymer deliver a higher average PCE of $9.3 \%$, due to improved $V_{\mathrm{OC}}, J_{\mathrm{SC}}$, and $F F$ values of $0.94 \mathrm{~V}, 15.9 \mathrm{~mA} \mathrm{~cm}^{-2}$, and $62 \%$, respectively (champion cell performance $9.8 \%$ ). We estimated the effective loss in $J_{\mathrm{SC}}$ as $1-J_{S C} / J_{\max }$, thin film, where $J_{\max \text {, thin film }}$ is the maximum available current density obtained for unity internal quantum efficiency (IQE) (Figure S2) determined by transfer matrix simulation (see Table 1). The associated short circuit 
current density loss of the three devices is $63 \%, 27 \%$, and $23 \%$ for PBDT[2HT]:ITIC, PBDT(T)[2H]T:ITIC, and PBDT(T)[2F]T:ITIC, respectively. The values of the $F F$ are comparable for the alkoxy and thienyl sidechain-substituted donor polymers, whilst improved significantly for the fluorinated donor polymer (62\%).

Table 1: Figures of Merit of PBDT[2HT]:ITIC, PBDT(T)[2H]T:ITIC, and PBDT(T)[2F]T:ITIC Solar Cells with a Blend Ratio of 1:1. The Standard Deviation was Determined across 15 Devices.

\begin{tabular}{ccccccc}
\hline BHJ system & $V_{\mathrm{OC}}$ & $J_{\mathrm{SC}}$ & $J_{\text {max, } \text { thin film }}$ & $F F$ & Avg. & Max. \\
& $(\mathrm{V})$ & $\left(\mathrm{mA} / \mathrm{cm}^{2}\right)$ & $\left(\mathrm{mA} / \mathrm{cm}^{2}\right)$ & $(\%)$ & PCE (\%) & PCE (\%) \\
\hline PBDT[2H]T & $0.77 \pm 0.02$ & $7.3 \pm 0.28$ & 18.9 & $41 \pm 1.2$ & $2.3 \pm 0.10$ & 2.5 \\
\hline PBDT(T)[2H]T & $0.72 \pm 0.01$ & $13.8 \pm 0.21$ & 19.7 & $40 \pm 0.6$ & $4.0 \pm 0.10$ & 4.2 \\
\hline PBDT(T)[2F]T & $0.94 \pm 0.01$ & $15.9 \pm 0.69$ & 20.4 & $62 \pm 1.0$ & $9.3 \pm 0.36$ & 9.8 \\
\hline
\end{tabular}




\section{ns- $\mu$ s Charge Carrier Recombination}

TA measurements were performed in the nanosecond-microsecond (ns- $\mu \mathrm{s})$ time range to monitor the charge carrier recombination dynamics following photoexcitation. In Figure 2, the timeintegrated TA spectra of all three blends are shown following optical excitation at $532 \mathrm{~nm}$, using a moderate laser fluence of $5.6 \mu \mathrm{J} / \mathrm{cm}^{2}$. The following spectral features are identified from the TA spectra in Figure 2a: ground state bleach (GSB) of the donor PBDT(T)[2F]T located within the spectral range of 2.0-2.6 eV, the ITIC acceptor ground state bleach spanning from 1.62-2.0 eV, and a broad photoinduced absorption (PA) covering the spectral region of $0.9-1.62 \mathrm{eV}$. Analogously, for PBDT(T)[2H]T:ITIC (Figure 2b) we assigned the spectral range of 1.1-1.6 eV to PA, whilst the GSB of the donor and acceptor are within the same range as mentioned above. Figure $2 \mathrm{c}$ shows the TA spectra of PBDT[2H]T:ITIC where the donor GSB is in the range of 2.15$2.6 \mathrm{eV}$, whilst the acceptor GSB is in the range of $1.6-2.0 \mathrm{eV}$, and the PA covers the spectral range of 0.94-1.6 eV. TA spectra of neat ITIC films are presented in Figure S8 for comparison. Accordingly, we assign the PA region to the predominant contribution from the respective donor polymer cations in all three cases. 

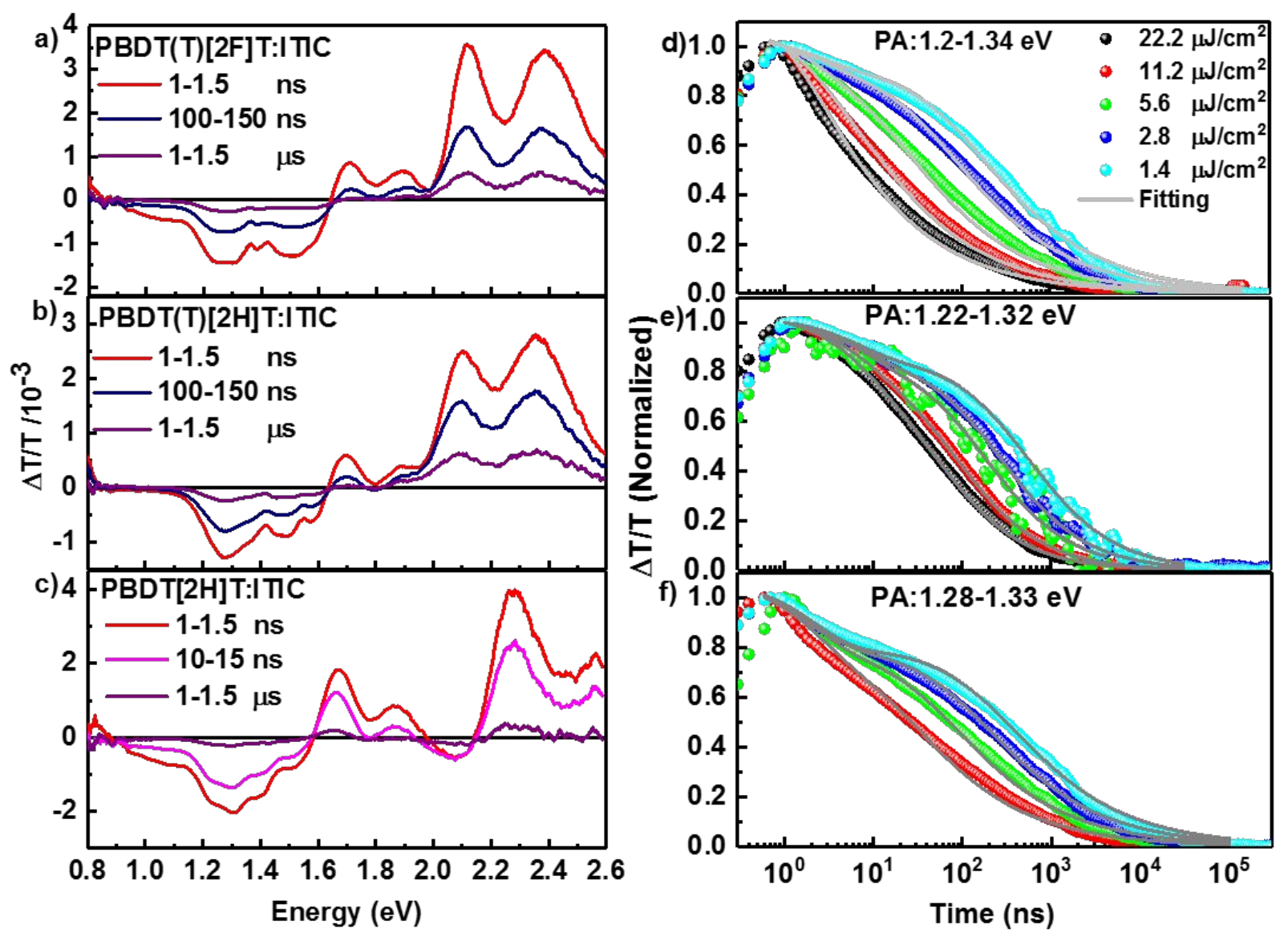

Figure 2: ns- $\mu \mathrm{s}$ TA spectra of BHJs following excitation at $532 \mathrm{~nm}$ with a fluence of $5.6 \mu \mathrm{J} / \mathrm{cm}^{2}$. a) PBDT(T)[2F]T:ITIC, b) PBDT(T)[2H]T:ITIC, and c) PBDT[2H]T:ITIC. The associated charge carrier dynamics tracked in the PA region of c) PBDT(T)[2F]T:ITIC, d) PBDT(T)[2H]T:ITIC, and e) PBDT[2H]T:ITIC.

Further evaluation of the charge recombination was performed by tracking the carrier dynamics in the PA region; results are shown in Figure 2d-f for a range of fluences. PBDT[2H]T:ITIC and PBDT(T)[2H]T:ITIC exhibit a component of fluence independent decay at early delay times, attributed to geminate recombination, most prominent in the first $10 \mathrm{~ns}$. Subsequently, fluence dependent carrier dynamics, assigned to non-geminate recombination, are observed at later times up to microseconds. In contrast, the PBDT(T)[2F]T:ITIC blend exhibits no obvious fluence independent decay component in the first ns after photoexcitation. A quantitative analysis was 
conducted by parameterizing the fluence dependent carrier dynamics based on the two-pool carrier recombination model. ${ }^{29}$ This model accounts for both geminate and non-geminate recombination processes, considered as two independent populations, however, spectrally indistinguishable. As demonstrated previously, under these conditions the charge carrier dynamics are described by the following equation:

$$
n(t)=N_{0}(1-f)\left[\exp \left(-k_{C T \rightarrow G S} t\right)\right]+\left[\lambda \gamma t+\left(f N_{0}\right)^{-\lambda}\right]^{-\frac{1}{\lambda}}
$$

Herein, $N_{0}$ is the total initial population (density) of charges, $f$ is the fraction of charges that undergo non-geminate recombination, $k_{C T \rightarrow G S}$ is the monomolecular (geminate) rate constant, $\lambda$ is the non-geminate recombination order, and $\gamma$ is the non-geminate coefficient, respectively. The $N_{0}$ values were determined from TDCF measurements as described in detail in the SI. Briefly, fluence dependent TDCF measurements using the same laser excitation as in ns- $\mu$ s TA were performed to determine the initial charge carrier densities of the respective systems as described previously; ${ }^{30}$ all $N_{0}$ values are given in Table S1. The measured TDCF transients of the respective systems can be found in Figures S12-14. The fitting parameters obtained from the global fit to equation (1) are summarized in Table 2; we deduce that the fraction of geminate recombination (1-f) is $20 \%$ for PBDT[2H]T:ITIC, $14 \%$ for PBDT(T)[2H]T:ITIC, and only $8 \%$ for PBDT(T)[2FT]:ITIC. However, these differences can only explain to some extent the different short circuit current density observed for the three systems. Equivalent bimolecular charge recombination coefficients $(\beta)$ were calculated (Table 3) from the fitting parameters for a carrier concentration 
approximately equivalent to that in devices under one sun illumination, i.e., $5 \times 10^{15} \mathrm{~cm}^{-3}$, using $\beta=\gamma_{\lambda} n_{1 \text { sun }}^{\lambda-1}$. Interestingly, the values are found to be comparable for the PBDT[2H]T- and PBDT(T)[2F]T-based blends, while an order of magnitude lower for the PBDT(T)[2H]T-based blend.

In the Langevin recombination model the bimolecular recombination coefficient $\beta$ is a function of the charge carrier mobilities. However, in organic BHJs the observed recombination coefficient is typically lower than the one predicted by Langevin theory (see also Table 3), calculated from the experimentally-determined charge carrier mobilities. Hence, typically a reduction factor $\zeta(\beta=\zeta$. $\left.\beta_{\text {Langevin }}\right)$ is introduced to account for the difference. Here, we hypothesize that the bimolecular recombination coefficient $\beta$ is lowest in PBDT(T)[2H]T:ITIC due to the combination of its low hole mobility (vide infra) and presence of bulky thienyl-sidechains, spatially separating the donor polymer from the ITIC acceptor, compared to the less bulky alkoxy-substituted donor polymer PBDT[2H]T. On the other hand, PBDT(T)[2F]T:ITIC exhibits a larger $\beta$, similar to PBDT[2H]T:ITIC, possibly due to its one order of magnitude higher hole mobility.

Table 2: Fit Parameters from Parameterization of Fluence-dependent Carrier Recombination Dynamcis using the Two-pool Charge Recombination Model. ${ }^{\mathrm{a}}$

\begin{tabular}{llll} 
Parameter & PBDT[2H]T:ITIC & PBDT(T)[2H]T:ITIC & PBDT(T)[2F]T:ITIC \\
\hline$f$ & $0.8 \pm 0.01$ & $0.86 \pm 0.01$ & $0.92 \pm 0.02$ \\
& & & \\
\hline
\end{tabular}




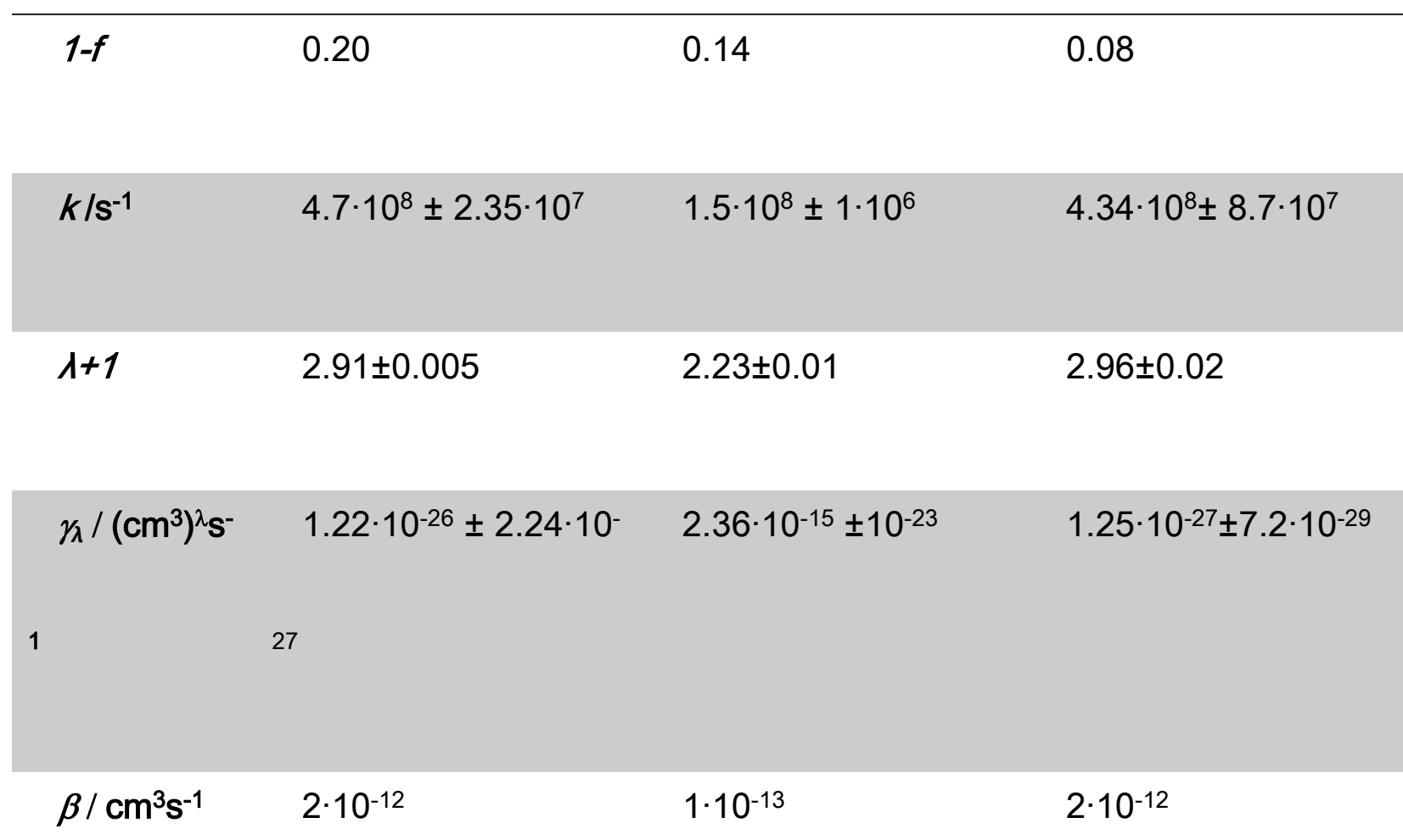

a The parameters are: $f$, the fraction of free charges undergoing non-geminate recombination, 1- $f$, the fraction of bound charges undergoing geminate recombination, $k$, the monomolecular (geminate) recombination rate constant, $1+1$, the non-geminate recombination order, $\gamma$, the non-geminate recombination coefficient, and $\beta$, the effective bimolecular recombination coefficient calculated for a carrier concentration approximately equivalent to that in devices under one sun illumination, i.e., $5 \times 10^{15} \mathrm{~cm}^{-3}$, using $\beta=\gamma_{\lambda} n_{1 \text { sun }}^{\lambda-1}$.

\section{Carrier Mobility and Field Dependence of Charge Generation}

In an attempt to determine the origin of the low $F F$, we used time-delayed collection field measurements to investigate the field dependence of charge generation and its impact on the device $F F$. Devices were photo-excited at $532 \mathrm{~nm}$ with a nanosecond laser pulse, while being kept at constant pre-bias. The voltage of the pre-bias was varied from - $1 \mathrm{~V}$ up to the respective open circuit voltage of the device. A collection bias of $-4 \mathrm{~V}$ was applied $10 \mathrm{~ns}$ after photoexcitation to ensure extraction of separated charge carriers prior to their (non-geminate) recombination. In order to reduce non-geminate recombination losses, the excitation fluence was kept low at $0.5 \mu \mathrm{J} / \mathrm{cm}^{2}$. We 
confirmed that this fluence is in the linear response regime by fluence-dependent measurements; the respective transients are displayed in Figures S12-14. Under these conditions, the total collected charge, $Q_{t o t}$, is a measure of the amount of free charges generated by the photoexcitation as a function of the applied pre-bias.

Figure 3 presents the total collected charge as measured by TDCF of three representative devices alongside their respective J-V-characteristics, more specifically, the total charge collected as a function of the applied pre-bias voltage, ranging here from $-1 \mathrm{~V}$ to $V_{O C}$. The PBDT[2HT]:ITIC device displayed weakly field-dependent charge generation, as indicated by the total collected charge $Q_{t o t}$ decreasing steadily towards the open circuit voltage. In contrast, both PBDT(T)[2FT]:ITIC and PBDT(T)[2H]T:ITIC systems yield a flat response of $Q_{t o t}$ vs. applied pre-bias, indicating entirely field independent charge generation. This implies that in the devices non-geminate recombination losses compete with charge extraction and thus determine the fill factor. We note that $Q_{t o t}$ from TDCF was scaled to match the device photocurrent at a bias of -1 V. Thus, the gap between $Q_{t o t}$ from TDCF and the experimentally-measured device J-V curve as shown in Figure 3 provides a measure of the relative non-geminate losses. Clearly, non-geminate recombination is less in PBDT(T)[2F]T:ITIC, while weakly field-dependent charge generation additionally reduces the fill factor of PBDT[2H]T:ITIC. Furthermore, non-geminate recombination losses are evident even at short circuit conditions for the PBDT[2H]T:ITIC system. In case of the PBDT(T)[2H]T:ITIC device, the fraction of non-geminate recombination is less than that of PBDT[2H]T:ITIC, while it is larger than that of PBDT(T)[2F]T:ITIC. Further indication of the competition between non-geminate recombination and carrier extraction is also provided by charge carrier mobility measurements (vide infra). ${ }^{31}$ We conclude, that weakly field-dependent charge generation is observed in PBDT[2H]T:ITIC, as $\mathrm{Q}_{\text {tot }}$ steadily decreases with applied pre- 
bias in TDCF experiments, whereas for the other two systems charge generation is entirely fieldindependent (compare solid black lines at $J_{S C}$ ), and that non-geminate charge recombination losses are significant in PBDT[2H]T:ITIC and PBDT(T)[2H]T:ITIC devices.

The bimolecular recombination coefficient was determined from both TA and TDCF experiments; both values are included in Table 3. In TDCF, the collected charge is measured as function of the time delay $t_{d e l}$ between photoexcitation and collection of charge carriers. Determination of the bimolecular recombination coefficient from TDCF measurements was done by fitting the quantity of collected charge, $Q_{c o l}$, as a function of $t_{d e l}$ using a model reported earlier by Kniepert and Albrecht et al. ${ }^{32-34}$ The TDCF transients and associated fits for all systems are presented in Figures S9-11.The values from TA and TDCF are of the same magnitude across all systems; however, TDCF consistently yields larger bimolecular recombination coefficients than TA, possibly due to the presence of carriers injected from the electrodes in TDCF experiments.

The carrier mobilities of two BHJ systems, namely PBDT[2H]T:ITIC and PBDT(T)[2F]T:ITIC, were measured by MIS-CELIV (metal-insulator-semiconductor charge extraction by linearly increasing voltage) and of the PBDT(T)[2H]T:ITIC system by SCLC (space-charge-limited current) measurements (Figure S15, Table S2). The hole mobilities were found to be: $3 \cdot 10^{-5} \mathrm{~cm}^{2} \mathrm{~V}^{-1} \mathrm{~s}^{-1}$ for PBDT(T)[2F]T:ITIC, $3.8 \cdot 10^{-6} \mathrm{~cm}^{2} \mathrm{~V}^{-1} \mathrm{~s}^{-1}$ for PBDT(T)[2H]T:ITIC, and $5.7 \cdot 10^{-6} \mathrm{~cm}^{2} \mathrm{~V}^{-1} \mathrm{~s}^{-1}$ for PBDT[2H]T:ITIC. Furthermore, the electron mobilities were found to be similar in all three systems: $1.2 \cdot 10^{-5} \mathrm{~cm}^{2} \mathrm{~V}^{-1} \mathrm{~s}^{-1}$ for PBDT(T)[2F]T:ITIC, $1.5 \cdot 10^{-5} \mathrm{~cm}^{2} \mathrm{~V}^{-1} \mathrm{~s}^{-1}$ for PBDT(T)[2H]T:ITIC, and $2.2 \cdot 10^{-5} \mathrm{~cm}^{2} \mathrm{~V}^{-1} \mathrm{~s}^{-1}$ for PBDT[2H]T:ITIC. Hence, the hole extraction is slower for the PBDT[2H]T:ITIC and PBDT(T)[2H]T:ITIC systems, as the hole mobility is about one order lower than in PBDT(T)[2F]T:ITIC. From the TDCF data analysis and mobility 
measurements, we conclude that non-geminate recombination losses compete with carrier extraction and limit the $F F$ and the PCE of PBDT[2H]T:ITIC devices. In contrast, the PBDT(T)[2F]T:ITIC exhibits significantly less non-geminate recombination, faster carrier extraction, and better balanced carrier mobilities, thus a significantly improved FF.

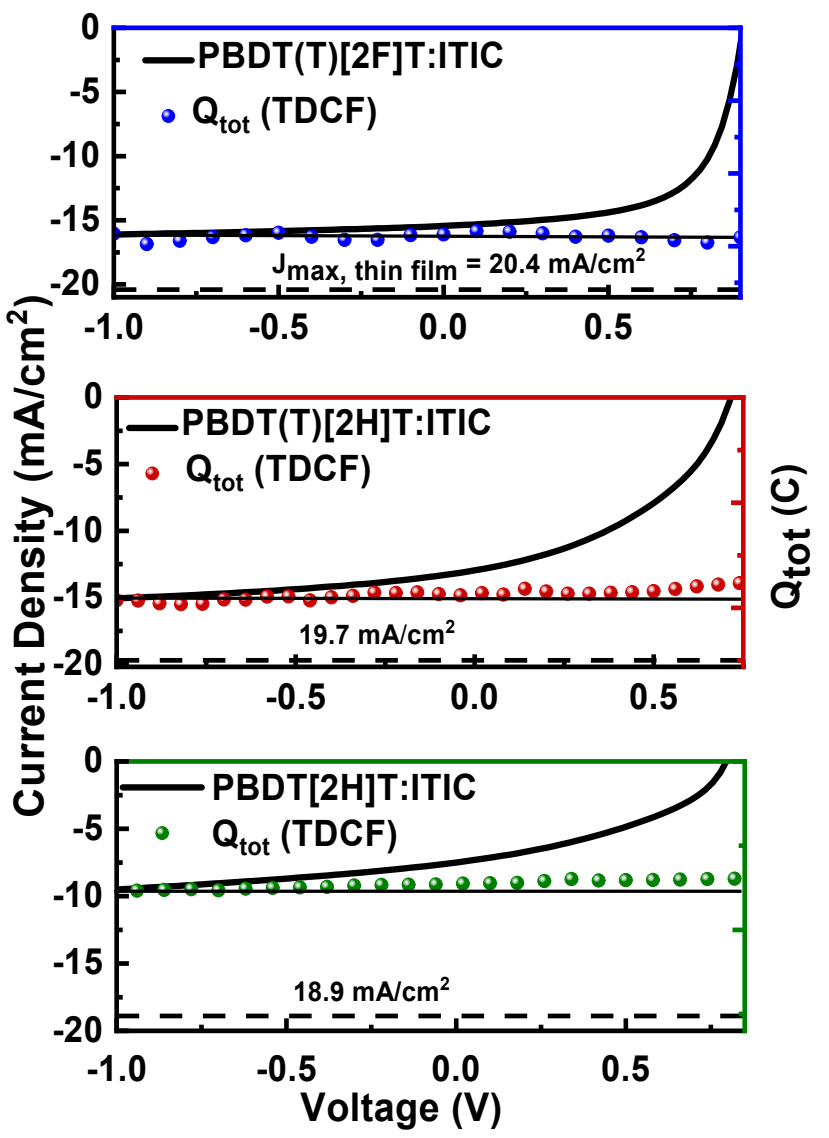

Figure 3: Total collected charge $Q_{t o t}(\mathrm{C})$ (filled dots) measured by pre-bias dependent TDCF experiments plotted along the experimentally-measured device JV-curves (solid lines) of the respective devices. $Q_{\text {tot }}$ was scaled to match the devices' photocurrent at a bias of $-1 \mathrm{~V}$. Note the weak field dependence of charge generation in PBDT[2H]T:ITIC and complete absence of field dependence in the other two systems. The horizontal dashed lines indicate the maximum available photocurrent based on the devices' active layer thickness, assuming 100\% IQE (see Figure S2).

\section{Simulation of Device J-V Characteristics}


Next, we simulated the J-V characteristics of the solar cells using Setfos 4.6 (FLUXiM AG), a commercial numerical drift-diffusion device simulation tool; further details about the software can be found in the respective literature. ${ }^{35}$

Table 3: Input Parameters ${ }^{\mathrm{a}}$ and Figures of Merit Obtained ${ }^{\mathrm{b}}$ by Simulation of the JV Characteristics of Polymer:ITIC Based Solar Cells in Inverted Device Structurec

\begin{tabular}{|c|c|c|c|c|c|c|c|c|c|c|}
\hline $\begin{array}{l}\text { Polymer donor } \\
\text { in ITIC blend }\end{array}$ & $\begin{array}{c}I Q E_{a v g} \\
(\%)\end{array}$ & $\begin{array}{c}\mu_{h} \\
\left(\mathrm{~cm}^{2} V^{-1} \mathbf{s}^{-1}\right)\end{array}$ & $\begin{array}{c}\mu_{e} \\
\left(\mathrm{~cm}^{2} V^{-1} \mathbf{s}^{-1}\right)\end{array}$ & $\begin{array}{c}\beta_{\text {Langevin }} \\
(\text { calc. }) \\
\left(\mathbf{1 0}^{-11} \mathrm{~cm}^{3} \mathbf{s}^{-1}\right) \\
\end{array}$ & $\begin{array}{c}\beta(\exp .) \\
\left(10^{-12} \mathrm{~cm}^{3} \mathrm{~s}^{-1}\right)\end{array}$ & $\begin{array}{c}\zeta=\beta / \\
\beta_{\text {Langevin }}\end{array}$ & $\begin{array}{c}J_{S C, \text { sim. }} \\
\left(\mathrm{mAcm}^{-2}\right)\end{array}$ & $\begin{array}{c}V_{O C}, \\
\text { sim } \\
(\mathrm{V}) \\
\end{array}$ & $\begin{array}{c}F F_{\text {sim }} \\
(\%)\end{array}$ & $\begin{array}{c}\text { PCE } \\
\text { sim } \\
(\%) \\
\end{array}$ \\
\hline \multirow[t]{2}{*}{ PBDT[2H]T } & 41 & $5.7 \times 10^{-6}$ & $2.2 \times 10^{-5}$ & 1.52 & 2 (TA) & $\begin{array}{l}\text { 0.132 } \\
\text { (TA) }\end{array}$ & 7.6 & 0.79 & 39 & 2.4 \\
\hline & & & & & 9 (TDCF) & $\begin{array}{l}0.593 \\
\text { (TDCF) }\end{array}$ & 6.1 & 0.76 & 35 & 1.6 \\
\hline \multirow[t]{2}{*}{ PBDT(T) $[2 \mathrm{H}] \mathrm{T}$} & 76 & $3.8 \times 10^{-6}$ & $1.5 \times 10^{-5}$ & 1.03 & 0.1 (TA) & $\begin{array}{l}\mathbf{0 . 0 0 9 7} \\
\text { (TA) }\end{array}$ & 13.4 & 0.73 & 41 & 4.0 \\
\hline & & & & & 0.5 (TDCF) & $\begin{array}{l}0.049 \\
\text { (TDCF) }\end{array}$ & 11.5 & 0.69 & 36 & 2.9 \\
\hline \multirow[t]{2}{*}{$\operatorname{PBDT}(\mathrm{T})[2 \mathrm{~F}] \mathrm{T}$} & 84 & $3.0 \times 10^{-5}$ & $1.2 \times 10^{-5}$ & 2.30 & 2 (TA) & $\begin{array}{l}0.0869 \\
\text { (TA) }\end{array}$ & 16.0 & 0.96 & 56 & 8.6 \\
\hline & & & & & 6 (TDCF) & $\begin{array}{l}0.261 \\
\text { (TDCF) }\end{array}$ & 15.0 & 0.93 & 48 & 6.8 \\
\hline
\end{tabular}

${ }^{\mathrm{a}}$ Experimentally-determined parameters, namely $\mathrm{IQE}_{\mathrm{avg}}(400-750 \mathrm{~nm}), \mu_{\mathrm{h}}, \mu_{\mathrm{e}}, \beta_{\text {Langevin }}, \beta$, and the recombination reduction factor $(\zeta)$, used as inputs to the simulation of the J-V curves.

${ }^{\mathrm{b}}$ Resulting (simulated) $J_{S C}, V_{O C}$, and $F F$ obtained by Setfos. Both the values of $\zeta$ from TA and TDCF experiments were used in the simulations for comparison.

${ }^{c}$ Device structure used in the simulation: glass $/ \mathrm{ITO}(120 \mathrm{~nm}) / \mathrm{ZnO}(30 \mathrm{~nm}) / \mathrm{BHJ}(80 \mathrm{~nm}) / \mathrm{MoO}_{3}(7 \mathrm{~nm}) / \mathrm{Ag}(100 \mathrm{~nm})$.

As input parameters, the charge carrier mobilities, average device internal quantum efficiency $\left(\mathrm{IQE}_{\mathrm{avg}}\right)$, and the bimolecular recombination coefficient $\beta$ determined from TA experiments were used in the simulation of the J-V curves. Furthermore, the simulations used the refractive index $(n)$ and extinction coefficient $(k)$ (see Figure S16) of the BHJ thin films obtained by spectroscopic ellipsometry measurements, the ionization energy (IE) and bandgap (Figure S6) determined by photoelectron spectroscopy in air (PESA) and from the intercept of the UV-Vis absorption and PL spectra of the materials added to the IE, respectively. The IQE values were determined from the external quantum efficiency (EQE) spectra, shown in Figure S3-S5, using: $I Q E=E Q E /(1$ Reflectance-Parasitic Absorptance). Reflectance spectra were collected in an integrating sphere using the same EQE system, while the parasitic absorption spectra were obtained from transfer 
matrix modelling. We note that the experimentally-determined reflectance of all three BHJ thin films is in good agreement with the simulated one as shown in Figure S16. More details of the simulation is provided in the SI.

Using the aforementioned parameters, the J-V curves of the solar cells were simulated as depicted in Figure 4. The obtained figures of merit, namely $J_{S C}, V_{O C}, F F$, and PCE, are displayed in Table 3. The simulated J-V curves are in good agreement with the experimentally-measured ones, yet the match is not absolute. The likely reason is the carrier concentration dependence of the bimolecular recombination coefficient $\beta$, as very recently discussed by us in another work. ${ }^{36}$ In short, as the carrier concentration in the device typically varies significantly between $V_{O C}$ and $J_{S C}$ conditions, a single $\beta$ value as used in the simulation, does not adequately fit the entire bias range, that is, carrier concentration range between $J_{S C}$ and $V_{O C}$.

We note also that $\mathrm{J}-\mathrm{V}$ curves simulated using $\beta$ determined by TDCF experiments as input yield a poor match with the experimentally-determined data (Figure S17); $J_{S C}$ and $F F$ are significantly underestimated. This has recently been discussed by us in our work on all-polymer BHJ films, which demonstrated that TDCF tends to overestimate $\beta \cdot{ }^{37}$ 


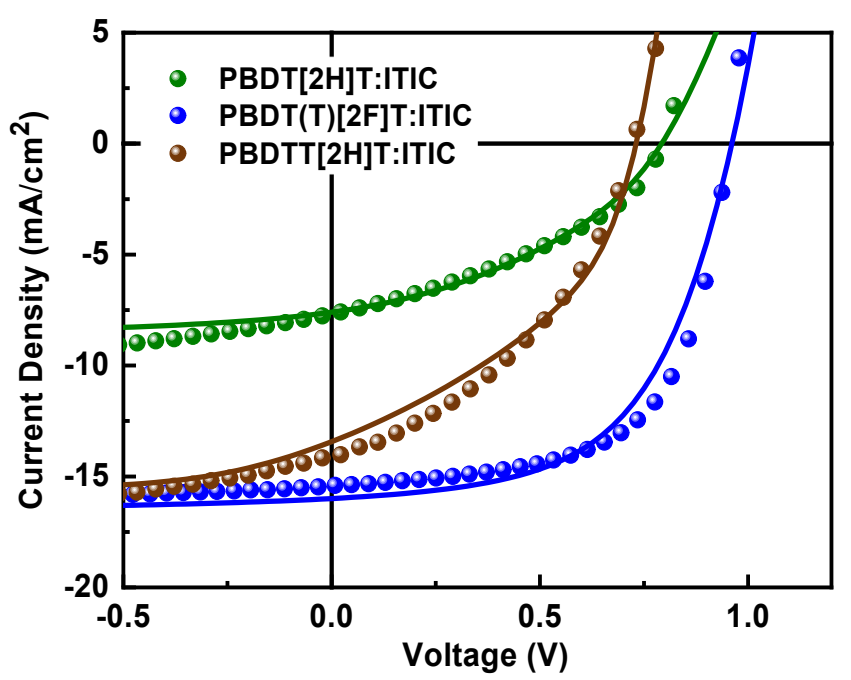

Figure 4: Simulated J-V characteristics (solid lines) obtained by Setfos drift-diffusion simulations using the experimentally-determined parameters and the non-geminate recombination coefficients from TA measurements (see Table 3). Underneath are the experimentally-measured device J-V characteristics (filled dots) of the three ITIC-based BHJ solar cell systems. Note the slight mismatch of the simulated and measured $\mathrm{J}-\mathrm{V}$ characteristics, explained further in the main text.

We do not have precise information about the interfacial donor polymer/ITIC acceptor arrangement and interfacial energetic landscape, which both are experimentally challenging to access. Hence, we can only hypothesize here that the origin of the improved charge separation efficiency and less non-geminate recombination in PBDT(T)[2F]T:ITIC could be the separation of the donor polymer backbone and non-fullerene acceptor ITIC. This separation can be increased in PBDT(T)[2F]T:ITIC blends due to the polymer backbone fluorination and steric hindrance imposed by the thienyl-sidechain, as recently also discussed by others. ${ }^{38,39}$ More precisely, larger separation raises the CT state energy and thereby reduces the charge separation barrier, in turn facilitating free charge generation and reducing geminate recombination, but eventually also reduces the exciton-to-CT state charge transfer rate, if the separation becomes too large. In contrast, the charge separation efficiency is reduced, if the donor-acceptor separation is smaller, as could be the case in the other two polymer:ITIC blends. However, we cannot exclude that different 
spatial donor-acceptor arrangement adds to the observed differences in geminate recombination

spatial donor-acceptor arrangement adds to the observed differences in geminate recombination 
and charge separation. Overall, the $J_{S C \text {, meas }}$ relative to the maximum theoretical available $J_{\max \text {, thin }}$ 
film, is $39 \%, 70 \%$, and $78 \%$ in PBDT[2H]T:ITIC, PBDT(T)[2H]T:ITIC, and PBDT(T)[2F]T:ITIC, 
while only $20 \%, 14 \%$, and $8 \%$ of the photocurrent loss is due to geminate charge recombination. 
The remaining losses, especially in the PBDT[2H]T-based device, are fractionally due to 
inefficient exciton quenching, as inferred from time-resolved photoluminescence (TR-PL) experiments (Figure S7). In fact, the TR-PL data indicates only moderate exciton quenching in PBDT[2H]T:ITIC: $46 \%$ when the donor polymer is excited at $490 \mathrm{~nm}$, which is slightly more than the IQE $(35 \%)$ at this wavelength. This indicates that the IQE is severely limited by exciton recombination in addition to the geminate recombination losses observed by TA spectroscopy (vide supra). On the contrary, the PBDT(T)[2H]T-based blend exhibits an acceptor (ITIC) exciton quenching efficiency of $92 \%$, while when exciting the donor polymer at $490 \mathrm{~nm}$ the PL was entirely quenched. Surprisingly, the PBDT(T)[2F]T-based blend exhibited a lower donor exciton quenching efficiency of $76 \%$ when excited at $490 \mathrm{~nm}$, yet the highest IQE is obtained in devices, pointing to negligible geminate recombination losses, as also confirmed by TA experiments (vide supra), but less efficient exciton dissociation, in line with an alleged larger donor-acceptor distance as discussed above. Lastly, in the case of PBDT(T)[2H]T:ITIC, severe losses due to non-geminate recombination occur at the maximum power point (MPP) as confirmed by TDCF experiments;

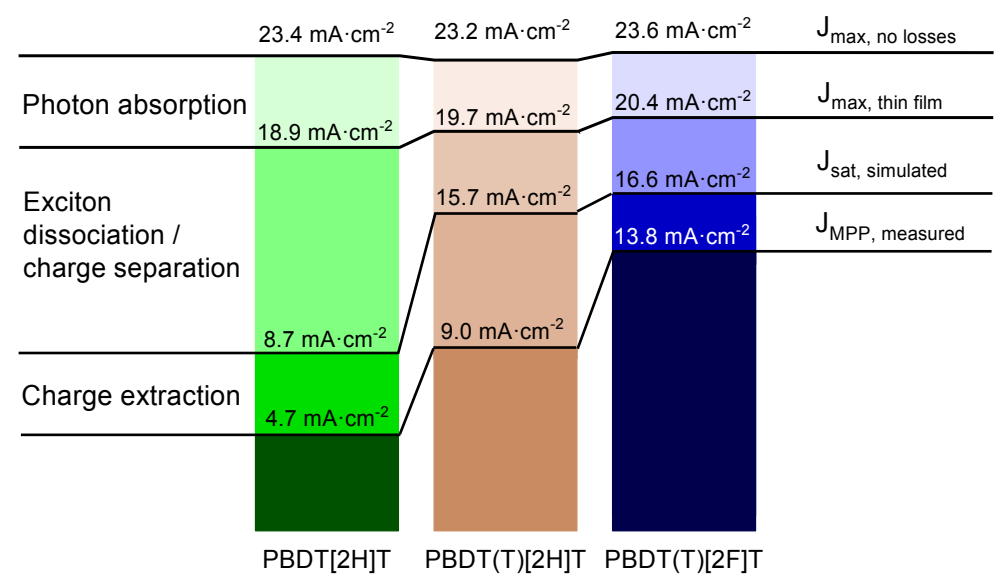

less for the thienyl sidechain substituted and backbone fluorinated PBDT(T)[2F]T donor polymer, of which the latter exhibits reduced non-geminate recombination losses. An overview of the fractional contribution of the individual loss processes to the device photocurrent is shown in Figure 5. 
Figure 5: Summary of photocurrent losses occurring in ITIC-based blends. $J_{\text {max, no losses }}$ denotes the calculated maximum available photocurrent in the absence of any losses. $J_{\text {max, thin film }}$ is the calculated current density for an IQE of $100 \%$ and for the experimentally-obtained optimal active layer thickness of $80 \mathrm{~nm}$. Furthermore, $J_{\text {sat, simulated }}$ is the simulated photocurrent accounting for incomplete exciton quenching and incomplete charge separation (geminate recombination), and $J_{M P P, \text { measured }}$ denotes the experimentally-measured photocurrent at the maximum power point (MPP, at a bias of $0.46 \mathrm{~V}, 0.52 \mathrm{~V}, 0.73 \mathrm{~V}$ from left to right), which accounts for losses due to carrier extraction, namely, non-geminate recombination.

\section{Conclusion}

In conclusion, we investigated the impact of alkoxy vs. thienyl substitution and backbone fluorination in three BDT-based donor polymers paired with the prototypical non-fullerene acceptor ITIC on the photophysics and device performance. Essentially, the combination of thienyl sidechains and fluorination of the polymer backbone leads to high short circuit current density, open circuit voltage, and fill factor in OPV devices. The origin of the difference in short circuit density was studied systematically by transient absorption, time-resolved PL, and time-delayed collection field experiments. TR-PL experiments demonstrated poor exciton quenching in the alkoxy-substituted system, PBDT[2H]T:ITIC; TA experiments demonstrated that geminate recombination losses are highest in PBDT[2H]T:ITIC, precisely $20 \%$, and lowest in the thienylsubstituted and fluorinated system, PBDT(T)[2F]T:ITIC, about $8 \%$. TDCF measurements revealed weakly field-dependent charge generation in PBDT[2H]T:ITIC and significant nongeminate recombination losses in the thienyl-substituted, non-fluorinated system, PBDT(T)[2H]T:ITIC, which both suffer from extraction losses due to their low hole mobility. On the other hand, entirely field-independent charge generation was observed in PBDT(T)[2F]Tbased devices in conjunction with improved charge carrier mobility. Consequently, the competition between carrier extraction and non-geminate recombination is shifted towards 
extraction, leading to improved fill factors. Lastly, J-V device characteristics were simulated using the experimentally-measured kinetic parameters, carrier mobilities, and average internal quantum efficiencies. The simulation was found to match the experimentally-measured J-V curves, supporting the relevance of spectroscopically-obtained parameters to describe processes in OPV devices under solar illumination corresponding to lower charge carrier densities, however, it also revealed the limitations of this approach.

\section{Supporting Information}

Steady-state absorption; Transfer matrix simulation of $J_{S C}$; Determination of internal quantum efficiency (IQE) spectra; Energy levels - Ionization energy (IE) and IE $+\mathrm{E}_{\mathrm{g}}\left(\mathrm{E}_{\mathrm{g}}\right.$ : optical gap); Timeresolved photoluminescence experiments; Transient absorption spectra of ITIC thin films; Calculation of initial carrier density $\mathrm{N}_{0}$ in TA experiments; Time-delayed collection field (TDCF) measurements; Charge carrier mobility measurements; SETFOS drift-diffusion simulation of JV curves.

\section{Acknowledgements}

This publication is based upon work supported by the King Abdullah University of Science and Technology (KAUST) Office of Sponsored Research (OSR) under Award No: OSR-2018CARF/CCF-3079. 


\section{References}

(1) Lin, Y.; Wang, J.; Zhang, Z.-G.; Bai, H.; Li, Y.; Zhu, D.; Zhan, X. An Electron Acceptor Challenging Fullerenes for Efficient Polymer Solar Cells, Adv. Mater. 201527 1170-1174.

(2) Li, S.; Ye, L.; Zhao, W.; Yan, H.; Yang, B.; Liu, D.; Li, W.; Ade, H.; Hou, J. A Wide Band-Gap Polymer with a Deep HOMO Level Enables 14.2\% Efficiency in Polymer Solar Cells, J. Am. Chem. Soc. $20181407159-7167$.

(3) Zheng, Z.; Hu, Q.; Zhang, S.; Zhang, D.; Wang, J.; Xie, S.; Wang, R.; Qin, Y.; Li, W.; Hong, L.; et al. A Highly Efficient Non-Fullerene Organic Solar Cell with a Fill Factor over 0.80 Enabled by a Fine-Tuned Hole-Transporting Layer, Adv. Mater. 2018301801801.

(4) Cui, Y.; Yao, H.; Zhang, J.; Zhang, T.; Wang, Y.; Hong, L.; Xian, K.; Xu, B.; Zhang, S.; Peng, J.; et al. Over 16\% Efficiency Organic Photovoltaic Cells Enabled by a Chlorinated Acceptor with Increased Open-circuit Voltages, Nat. Commun. 2019102515.

(5) Lin, Y.; Adilbekova, B.; Firdaus, Y.; Yengel, E.; Faber, H.; Sajjad, M.; Zheng, X.; Yarali, E.; Seitkhan, A.; Bakr, O. M.; et al. 17\% Efficient Organic Solar Cells Based on Liquid Exfoliated $\mathrm{WS}_{2}$ as a Replacement for PEDOT:PSS, Adv. Mater. 2019311902965.

(6) Fan, B.; Zhang, D.; Li, M.; Zhong, W.; Zeng, Z.; Ying, L.; Huang, F.; Cao, Y. Achieving over 16\% Efficiency for Single-Junction Organic Solar Cells, Science China Chem. 201962 746752.

(7) Jiang, K.; Wei, Q.; Lai, J. Y. L.; Peng, Z.; Kim, H. K.; Yuan, J.; Ye, L.; Ade, H.; Zou, Y.; Yan, H. Alkyl Chain Tuning of Small Molecule Acceptors for Efficient Organic Solar Cells, Joule 20193 3020-3033.

(8) Yuan, J.; Zhang, Y.; Zhou, L.; Zhang, G.; Yip, H.-L.; Lau, T.-K.; Lu, X.; Zhu, C.; Peng, H.; Johnson, P. A.; et al. Single-Junction Organic Solar Cell with over 15\% Efficiency Using Fused-Ring Acceptor with Electron-Deficient Core, Joule 20193 1140-1151.

(9) Liu, Q.; Jiang, Y.; Jin, K.; Qin, J.; Xu, J.; Li, W.; Xiong, J.; Liu, J.; Xiao, Z.; Sun, K.; et al.18\% Efficiency organic solar cells. Sci. Bull. 2020, 65, 272-275.

(10) Firdaus, Y.; Maffei, L. P.; Cruciani, F.; Müller, M. A.; Liu, S.; Lopatin, S.; Wehbe, N.; Ndjawa, G. O. N.; Amassian, A.; Laquai, F.; et al. Polymer Main-Chain Substitution Effects on the Efficiency of Nonfullerene BHJ Solar Cells, Adv. Energy Mater. 201771700834.

(11) Price, S. C.; Stuart, A. C.; Yang, L.; Zhou, H.; You, W. Fluorine Substituted Conjugated Polymer of Medium Band Gap Yields 7\% Efficiency in Polymer-Fullerene Solar Cells, J. Amer. Chem. Soc. 2011133 4625-4631.

(12) Bronstein, H.; Frost, J. M.; Hadipour, A.; Kim, Y.; Nielsen, C. B.; Ashraf, R. S.; Rand, B. P.; Watkins, S.; McCulloch, I. Effect of Fluorination on the Properties of a Donor-Acceptor Copolymer for Use in Photovoltaic Cells and Transistors, Chem. of Mater. 201325 277-285.

(13) Liu, P.; Zhang, K.; Liu, F.; Jin, Y.; Liu, S.; Russell, T. P.; Yip, H.-L.; Huang, F.; Cao, Y., Effect of Fluorine Content in Thienothiophene-Benzodithiophene Copolymers on the Morphology and Performance of Polymer Solar Cells, Chem. of Mater. 201426 3009-3017.

(14) Wang, Y.; Xin, X.; Lu, Y.; Xiao, T.; Xu, X.; Zhao, N.; Hu, X.; Ong, B. S.; Ng, S. C. Substituent Effects on Physical and Photovoltaic Properties of 5,6Difluorobenzo[c][1,2,5]thiadiazole-Based D-A Polymers: Toward a Donor Design for High Performance Polymer Solar Cells, Macromolecules 201346 9587-9592.

(15) Warnan, J.; El Labban, A.; Cabanetos, C.; Hoke, E. T.; Shukla, P. K.; Risko, C.; Brédas, J.-L.; McGehee, M. D.; Beaujuge, P. M. Ring Substituents Mediate the Morphology of PBDTTPD-PCBM Bulk-Heterojunction Solar Cells, Chem. of Mater. 201426 2299-2306. 
(16) Chen, M.; Liu, D.; Li, W.; Gurney, R. S.; Li, D.; Cai, J.; Spooner, E. L. K.; Kilbride, R. C.; McGettrick, J. D.; Watson, T. M.; et al. Influences of Non-fullerene Acceptor Fluorination on Three-Dimensional Morphology and Photovoltaic Properties of Organic Solar Cells, ACS Appl. Mater. Interfaces 201911 26194-26203.

(17) Bauer, N.; Zhang, Q.; Rech, J. J.; Dai, S.; Peng, Z.; Ade, H.; Wang, J.; Zhan, X.; You, W. The Impact of Fluorination on Both Donor Polymer and Non-fullerene Acceptor: The More Fluorine, The Merrier, Nano Res. 201912 2400-2405.

(18) Du, X.; Heumueller, T.; Gruber, W.; Classen, A.; Unruh, T.; Li, N.; Brabec, C. J. Efficient Polymer Solar Cells Based on Non-fullerene Acceptors with Potential Device Lifetime Approaching 10 Years, Joule 20193 215-226.

(19) Du, Z.; Bao, X.; Li, Y.; Liu, D.; Wang, J.; Yang, C.; Wimmer, R.; Städe, L. W.; Yang, R.; Yu, D. Balancing High Open Circuit Voltage over 1.0 V and High Short Circuit Current in Benzodithiophene-Based Polymer Solar Cells with Low Energy Loss: A Synergistic Effect of Fluorination and Alkylthiolation, Adv. Ener. Mater. 201881701471.

(20) Jia, X. e.; Liu, G.; Chen, S.; Li, Z.; Wang, Z.; Yin, Q.; Yip, H.-L.; Yang, C.; Duan, C.; Huang, F.; et al. Backbone Fluorination of Polythiophenes Improves Device Performance of NonFullerene Polymer Solar Cells, ACS Appl. Energy Mater. 20192 7572-7583.

(21) Hu, Z.; Haws, R. T.; Fei, Z.; Boufflet, P.; Heeney, M.; Rossky, P. J.; Vanden Bout, D. A. Impact of Backbone Fluorination on Nanoscale Morphology and Excitonic Coupling in Polythiophenes, Proc. Nat. Acad. of Sci. 2017114 5113-5118.

(22) Leclerc, N.; Chávez, P.; Ibraikulov, O. A.; Heiser, T.; Lévêque, P. Impact of Backbone Fluorination on $\pi$-Conjugated Polymers in Organic Photovoltaic Devices: A Review, Polymers (Basel), 20161.

(23) Do, K.; Saleem, Q.; Ravva, M. K.; Cruciani, F.; Kan, Z.; Wolf, J.; Hansen, M. R.; Beaujuge, P. M.; Brédas, J.-L. Impact of Fluorine Substituents on $\pi$-Conjugated Polymer Main-Chain Conformations, Packing, and Electronic Couplings, Adv. Mater. 201628 8197-8205.

(24) Stuart, A. C.; Tumbleston, J. R.; Zhou, H.; Li, W.; Liu, S.; Ade, H.; You, W. Fluorine Substituents Reduce Charge Recombination and Drive Structure and Morphology Development in Polymer Solar Cells, J. Am. Chem. Soc. 2013135 1806-1815.

(25) Albrecht, S.; Janietz, S.; Schindler, W.; Frisch, J.; Kurpiers, J.; Kniepert, J.; Inal, S.; Pingel, P.; Fostiropoulos, K.; Koch, N.; et al. Fluorinated Copolymer PCPDTBT with Enhanced Open-Circuit Voltage and Reduced Recombination for Highly Efficient Polymer Solar Cells, $J$. Am. Chem. Soc. 2012134 14932-14944.

(26) Huo, L.; Zhang, S.; Guo, X.; Xu, F.; Li, Y.; Hou, J. Replacing Alkoxy Groups with Alkylthienyl Groups: A Feasible Approach To Improve the Properties of Photovoltaic Polymers, Angew. Chem. Int. Ed. $2011509697-9702$.

(27) Duan, R.; Ye, L.; Guo, X.; Huang, Y.; Wang, P.; Zhang, S.; Zhang, J.; Huo, L.; Hou, J. Application of Two-Dimensional Conjugated Benzo[1,2-b:4,5- $\mathrm{b}^{\prime}$ ]dithiophene in QuinoxalineBased Photovoltaic Polymers, Macromolecules 201245 3032-3038.

(28) Alamoudi, M. A.; Khan, J. I.; Firdaus, Y.; Wang, K.; Andrienko, D.; Beaujuge, P. M.; Laquai, F. Impact of Nonfullerene Acceptor Core Structure on the Photophysics and Efficiency of Polymer Solar Cells, ACS Energy Lett. 20183 802-811.

(29) Howard, I. A.; Mauer, R.; Meister, M.; Laquai, F. Effect of Morphology on Ultrafast Free Carrier Generation in Polythiophene:Fullerene Organic Solar Cells, J. Am. Chem. Soc. 2010132 14866-14876. 
(30) Khan, J. I; Ashraf, R. S.; Alamoudi, M. A.; Nabi, M. N.; Mohammed, H. N.; Wadsworth, A.; Firdaus, Y.; Zhang, W.; Anthopoulos, T. D.; McCulloch, I.; et al. P3HT Molecular Weight Determines the Performance of P3HT:O-IDTBR Solar Cells, Sol. RRL 201931900023.

(31) Bartesaghi, D.; Pérez, I. d. C.; Kniepert, J.; Roland, S.; Turbiez, M.; Neher, D.; Koster, L. J. A. Competition between Recombination and Extraction of Free Charges determines The Fill Factor of Organic Solar Cells. Nat. Commun. 201567083.

(32) Albrecht, S.; Schindler, W.; Kurpiers, J.; Kniepert, J.; Blakesley, J. C.; Dumsch, I.; Allard, S.; Fostiropoulos, K.; Scherf, U.; Neher, D. On the Field Dependence of Free Charge Carrier Generation and Recombination in Blends of PCPDTBT/PC70BM: Influence of Solvent Additives, J. Phys. Chem. Lett. 20123 640-645.

(33) Kniepert, J.; Lange, I.; van der Kaap, N. J.; Koster, L. J. A.; Neher, D. A Conclusive View on Charge Generation, Recombination, and Extraction in As-Prepared and Annealed P3HT:PCBM Blends: Combined Experimental and Simulation Work, Adv. Energy Mater. 201441301401.

(34) Kniepert, J.; Schubert, M.; Blakesley, J. C.; Neher, D. Photogeneration and Recombination in P3HT/PCBM Solar Cells Probed by Time-Delayed Collection Field Experiments Photogeneration and Recombination in P3HT/PCBM Solar Cells Probed by Time-Delayed Collection Field Experiments, J. Phys. Chem. Lett. 20112 700-705.

(35) Firdaus, Y.; Le Corre, V. M.; Khan, J. I.; Kan, Z.; Laquai, F.; Beaujuge, P. M.; Anthopoulos, T. D. Key Parameters Requirements for Non-Fullerene-Based Organic Solar Cells with Power Conversion Efficiency $>20 \%$ Key Parameters Requirements for Non-Fullerene-Based Organic Solar Cells with Power Conversion Efficiency >20\%, Adv. Sci. 201961802028.

(36) Karuthedath, S.; Gorenflot, J.; Melianas, A.; Kan, Z.; Kemerink, M.; Laquai, F. Buildup of Triplet-State Population in Operating TQ1:PC71BM Devices Does Not Limit Their Performance. J. Phys. Chem. Lett. 2020, 2838-2845.

(37) Karuthedath, S.; Melianas, A.; Kan, Z.; Pranculis, V.; Wohlfahrt, M.; Khan, J. I.; Gorenflot, J.; Xia, Y.; Inganäs, O.; Gulbinas, V.; et al. Thermal annealing reduces geminate recombination in TQ1:N2200 all-polymer solar cells. J. Mater. Chem. A 2018, 6 7428-7438.

(38) Holcombe, T. W.; Norton, J. E.; Rivnay, J.; Woo, C. H.; Goris, L.; Piliego, C.; Griffini, G.; Sellinger, A.; Brédas, J.-L.; Salleo, A.; et al. Steric Control of the Donor/Acceptor Interface: Implications in Organic Photovoltaic Charge Generation, J. Am. Chem. Soc. $201113312106-$ 12114.

(39) Deshmukh, K. D.; Prasad, S. K. K.; Chandrasekaran, N.; Liu, A. C. Y.; Gann, E.; Thomsen, L.; Kabra, D.; Hodgkiss, J. M.; McNeill, C. R. Critical Role of Pendant Group Substitution on the Performance of Efficient All-Polymer Solar Cells, Chem. Mater. 2017 29 804-816. 
TOC Figure

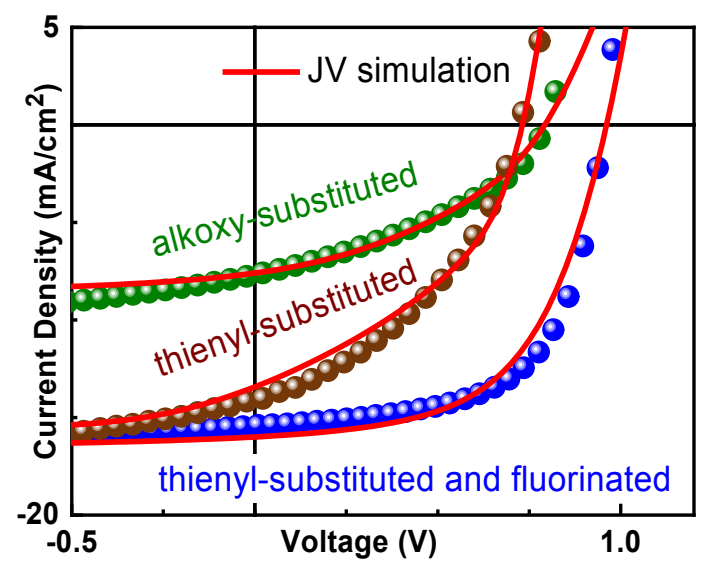

